\title{
Image-guided high-dose-rate interstitial brachytherapy - a valuable salvage treatment approach for loco-regional recurrence of papillary thyroid cancer
}

\author{
Ning Wu, MD*, Hongfu Zhao, MS*, Dongmei Han, BS, Guanghui Cheng, MD, PhD, Zhipeng Zhao, MS, Yuxin Ge, BS \\ *These two authors contributed equally to this work. \\ Department of Radiation Oncology, China-Japan Union Hospital of Jilin University, Changchun, China
}

\begin{abstract}
Purpose: To report the treatment effect of image-guided high-dose-rate (HDR) interstitial brachytherapy for refractory recurrence of papillary thyroid cancer (PTC).

Case report: This 66-year-old female presented with recurrence 5 years after thyroidectomy for PTC. Despite external irradiation and radioactive ${ }^{131} \mathrm{I}$, the lesion expanded as $3.7 \times 3.0 \times 2.3 \mathrm{~cm}^{3}$ and $2.0 \times 1.5 \times 1.5 \mathrm{~cm}^{3}$. The locoregional recurrent tumor was treated with image-guided HDR interstitial brachytherapy. The total dose of 30 Gy in 6 fractions were delivered on the whole recurrent tumor.

Results: Removal of the recurrent tumor was securely achieved by HDR interstitial brachytherapy guided with ultrasound, computed tomography (CT), and magnetic resonance imaging (MRI) scanning. The refractory tumor in the patients healed uneventfully after HDR interstitial brachytherapy without recurrence during the 14 months of follow-up.

Conclusions: The image-guided HDR interstitial brachytherapy may be a valuable salvage treatment approach for refractory recurrence of PTC.

J Contemp Brachytherapy 2016; 8, 2: 150-155 DOI: 10.5114/jcb.2016.59127
\end{abstract}

Key words: papillary thyroid cancer, HDR interstitial brachytherapy, local recurrence.

\section{Purpose}

Thyroid cancers are mainly papillary, follicular, medullary, or anaplastic thyroid cancer [1]. Based on report from the National Cancer Institute (NCI), more than $80 \%$ of people with thyroid cancer in the United States have papillary type of thyroid cancer. It begins in follicular cells of the thyroid and usually grows slowly. If early clinically determined, most people with papillary thyroid cancer (PTC) can be cured successfully [2]. Surgery remains the mainstay of treatment for PTC. The most effective management of aggressive thyroid cancers is surgical removal of thyroid gland (thyroidectomy) followed by radioactive iodine ablation and thyroid-stimulating hormone (TSH) suppression therapy [3, 4]. After successful treatment, 35 percent of the patients may experience recurrence [5].

Loco-regional recurrence in patients with PTC should undergo surgical resection of the local recurrence, radioactive ${ }^{131}$ I therapy, and involved-field radiotherapy, if it may be safely administered. External irradiation is not routinely used to treat thyroid cancer. But it may be used for the thyroid cancer that is unresectable or has spread to other parts of the body, as well as ${ }^{131}$ I for papillary/follicular thyroid cancer that cannot be completely removed with surgery, or to shrink any type of thyroid cancer that has come back after it was first treated. The therapeutic researches emphasized the importance of individualizing treatment strategies in patient with a recurrence of disease limited to a local site [4]. In this context, we propose a valuable salvage technique using image-guided highdose-rate (HDR) interstitial brachytherapy to treat refractory recurrence of PTC after conventional treatment.

\section{Case report}

\section{Patient presentation}

The case of a 66-year-old female with recurrence of PTC after initial surgery and radioactive ${ }^{131}$ I therapy combined with external beam radiotherapy is presented. Six years before admitting to our clinic, she visited a vicinity clinic with a hard nodule in the thyroid region of the left 
neck, which was found during a physical examination. The lump $\left(3.8 \times 2.5 \times 2.0 \mathrm{~cm}^{3}\right)$, which located in the left lobe of thyroid has grown into the laryngeal cartilage and the metastatic tumor cells was found in left submandibular lymph nodes. She received thyroidectomy and dissection of central neck compartment; pathological result revealed papillary carcinoma. She was diagnosed with PTC (T4aN1bM0; IVa stage) and accepted the TSH-suppression therapy with taking $150 \mu \mathrm{g} /$ day dose of levothyroxine (L-T4) immediately. One year after the initial thyroidecto$\mathrm{my}$, the patient underwent locoregional surgery again for the left neck recurrence $\left(3.0 \times 3.0 \times 2.0 \mathrm{~cm}^{3}\right)$. Then radioactive ${ }^{131} \mathrm{I}$ was used for ablation of residual thyroid tissue after surgery. She received the external beam radiotherapy with a total of $54 \mathrm{~Gy}$ in $2 \mathrm{~Gy}$ fractions for the left neck and $40 \mathrm{~Gy}$ in 2 Gy fractions for the other side of neck. Three years later, the recurrent tumor $\left(5.0 \times 4.5 \times 3.0 \mathrm{~cm}^{3}\right)$ in the ipsilateral neck was diagnosed and removed by the third time surgery. Postoperative pathology confirmed invasion of PTC. The patient's condition remained stable for one year until a complaint of oppressive pain with a stony hard mass with serious skin fibrosis in the left neck in 2014. She had an abnormal MRI scan revealing an area of distortion of approximately $3.7 \times 3.0 \times 2.3 \mathrm{~cm}^{3}$ and $2.0 \times 1.5 \times 1.5 \mathrm{~cm}^{3}$ in size (see Figure 1). Despite recommendation for subsequent palliative resection, the patient refused surgery. She was referred to our clinic for treatment and accepted the suggestion of brachytherapy.

\section{Treatment modalities}

Before processing each treatment, informed consent was obtained from the patient. Treatments were performed with standard institutional approval. High-dose-rate interstitial brachytherapy of 30 Gy in 6 fractions during 3 days (5 Gy each fraction, twice a day with 6 hours interval, 3 days) was administered to the patient. OncoSmart ProGuide needles (Nucletron, an Elekta company, Elekta $\mathrm{AB}$, Stockholm, Sweden) were used and the treatment was

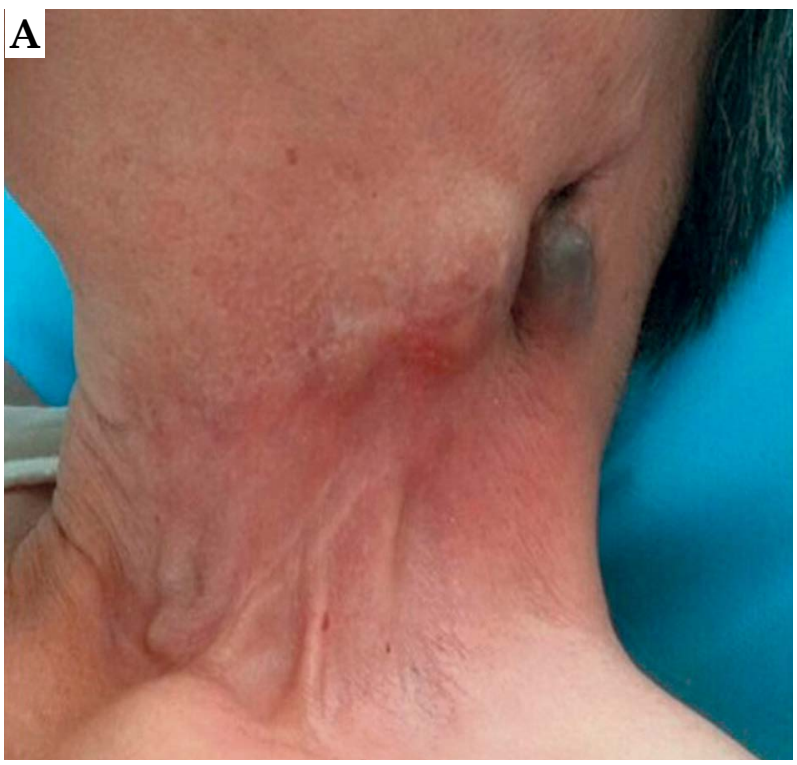

Fig. 1. Recurrent tumor in the ipsilateral neck performed under anesthesia. Electrocardiogram, arterial oxygen pressure, respiration, and blood pressure monitoring were performed during the procedure. Five applicator needles (1.1 $\mathrm{mm}$ in external diameter and $20 \mathrm{~cm}$ in length) were inserted to the target under image guidance, with interval of $1.0 \mathrm{~cm}$ to make sure the adequate dose distribution and target volume coverage (see Figure 2). OncoSmart ProGuide CT-Markers were put inside the catheters in order to facilitate their reconstruction. After implanting the applicators, fine-pitch $(2 \mathrm{~mm}) \mathrm{X}$-ray CT images were then acquired and transferred to the treatment planning computer (see Figure 3). The CT-imaging data combined with the MRI before interstitial brachytherapy were used to contour the gross target volume (GTV) and the clinical target volume (CTV). The CTV was expanded from GTV by $10 \mathrm{~mm}$ and restricted by the volume of critical organs. A CT-based treatment plan was created using a graphic optimization tool (Treatment Planning System Oncentra V4.3, Nucletron, an Elekta company, Elekta AB, Stockholm, Sweden). The normalization and optimization to the target volume was performed.

The dose volume histogram (DVH) parameters are recommended for the evaluation of target volume and organs at risk (OARs). The dose distribution to target volume and OARs were showed in Figure 4. In the brachytherapy plan, 30 Gy was prescribed to $100 \%$ of the target volume. $\mathrm{D}_{\max }$ (the maximum dose) of the spinal cord and left intervertebral vessel, $D_{\text {mean }}$ (the mean dose) of larynx, left parotid gland, and hypopharynx were mainly considered. The equivalent dose for a 2 Gy fraction schedule was calculated using the EQD2 model, at $\alpha / \beta=3\left(\mathrm{~Gy}_{\mathrm{EQD} 2, \alpha / \beta=3}\right)$ for the OARs and $\alpha / \beta=10\left(\mathrm{~Gy}_{\mathrm{EQD} 2, \alpha / \beta=10}\right)$ for the target. Dose volume histogram parameters were analyzed taking into account the volume ratio of the target receiving $90 \%$ or $150 \%$ of the therapeutic dose $\left(\mathrm{V}_{90 \%}\right.$ and $\mathrm{V}_{150 \%}$, respectively), the equivalent dose delivered to $98 \%, 90 \%$ and $50 \%$ of the target volume $\left(\mathrm{D}_{98 \%}, \mathrm{D}_{90 \%}\right.$, and $\mathrm{D}_{50 \%}$, respectively), the maximum equivalent dose delivered to spinal

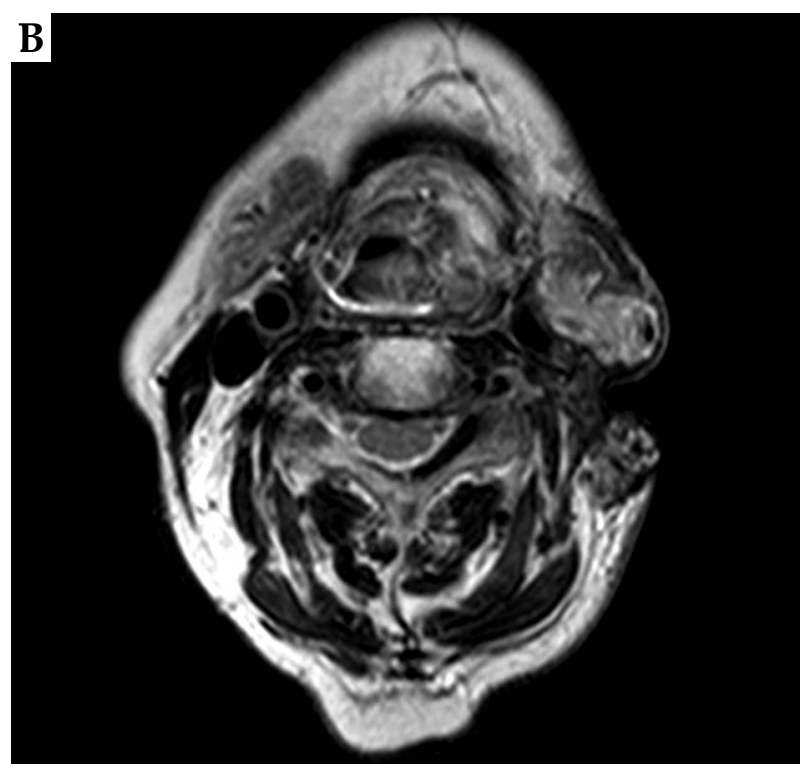



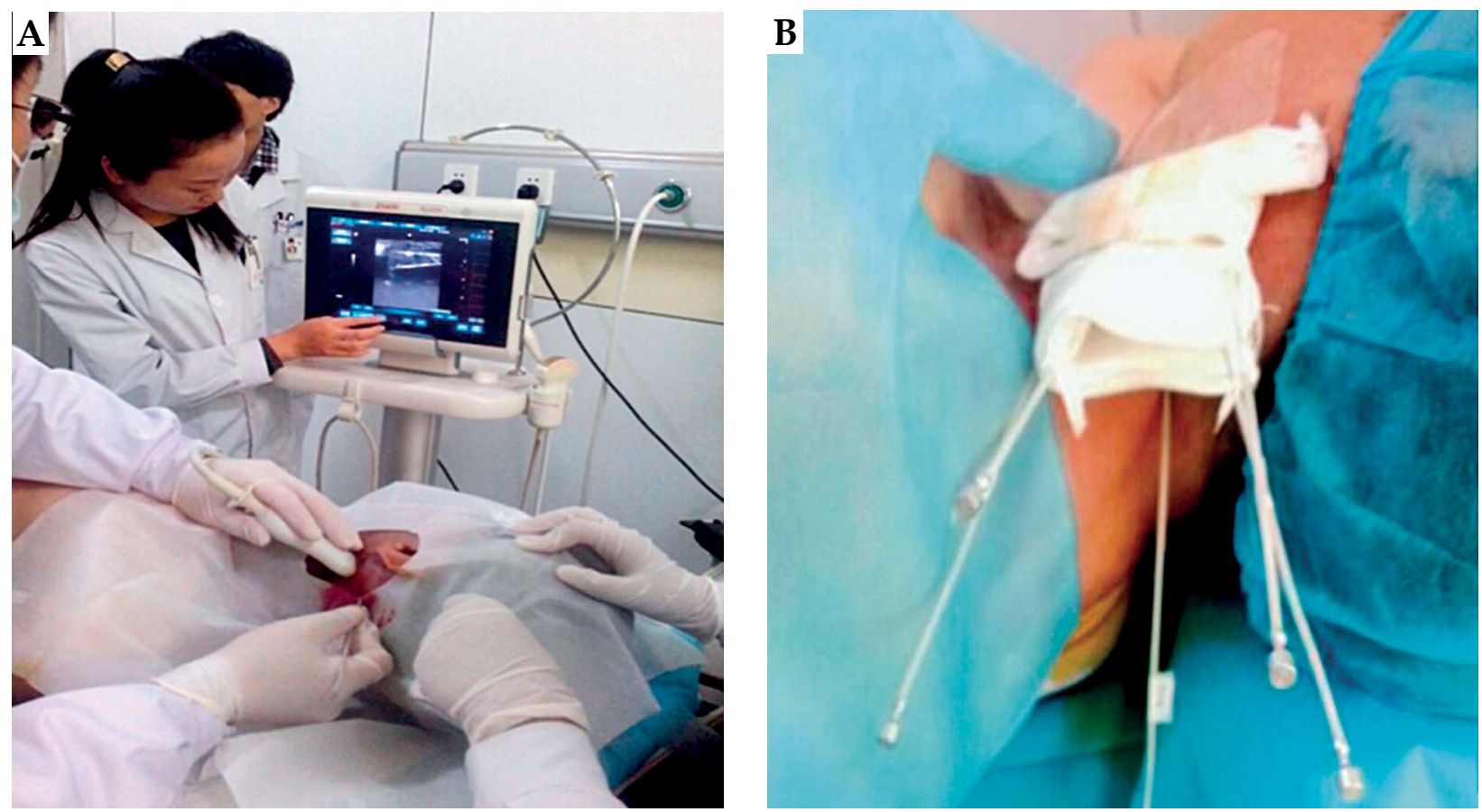

Fig. 2. Five applicator needles inserted to the target under ultrasound guidance
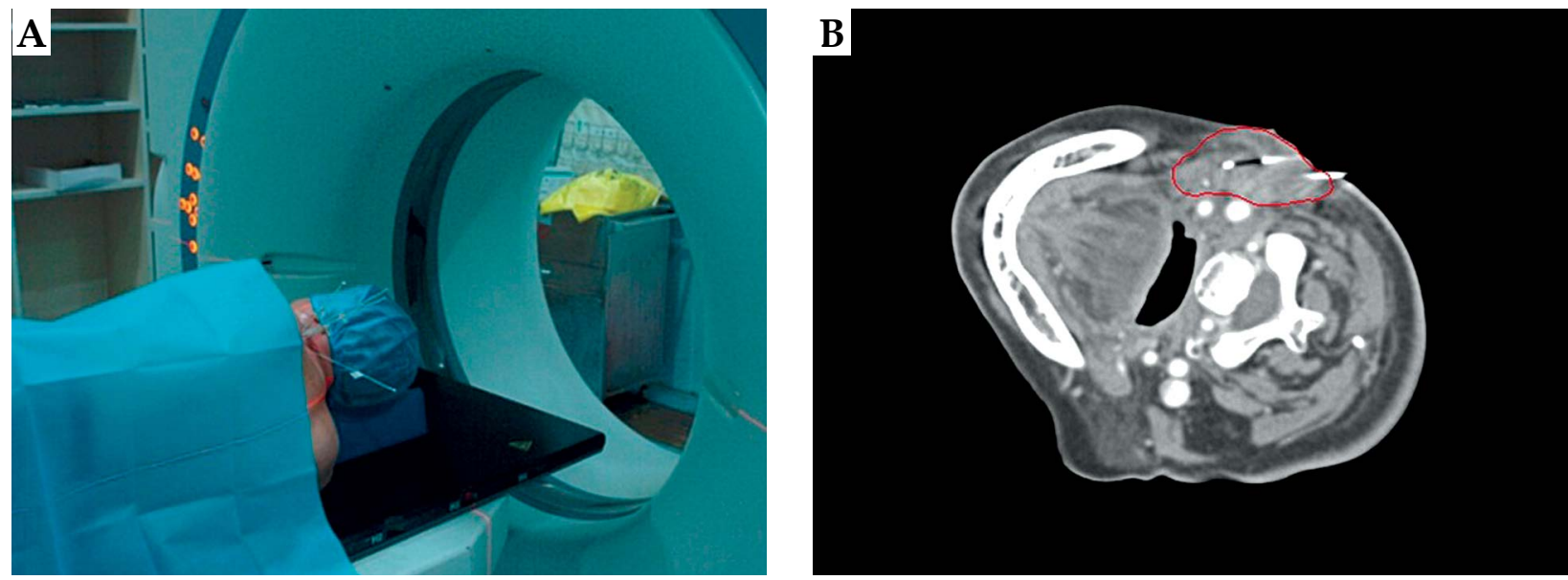

Fig. 3. The position of applicator needles in computed tomography images

cord and left intervertebral vessel $\left(\mathrm{D}_{\text {max-spinal cord }}\right.$ and $\mathrm{D}_{\text {max- }}$ left intervertebral vessel), the mean equivalent dose delivered to larynx, left parotid gland, and hypopharynx $\left(\mathrm{D}_{\text {mean-larynx }}\right.$ $\mathrm{D}_{\text {mean-left parotid gland }}$ and $\mathrm{D}_{\text {mean-hypopharynx }}$ ). The following values were accepted: below $\mathrm{V}_{90 \%}$ was $4.2 \%, \mathrm{~V}_{150 \%}$ was $58.1 \% ; \mathrm{D}_{98 \%}$ was $28.2 \mathrm{~Gy}, \mathrm{D}_{90 \%}$ was $39.0 \mathrm{~Gy}, \mathrm{D}_{50 \%}$ was 75.0 Gy; $\mathrm{D}_{\text {max-spinal cord }}$ was $7.8 \mathrm{~Gy}$; $\mathrm{D}_{\text {max-left intervertebral vessel }}$ was $25.2 \mathrm{~Gy} ; \mathrm{D}_{\text {mean-larynx }}$ was $8.4 \mathrm{~Gy} ; \mathrm{D}_{\text {mean-left parotid gland }}$ was $5.4 \mathrm{~Gy}$; $\mathrm{D}_{\text {mean-hypopharynx }}$ was $6.6 \mathrm{~Gy}$.

After transporting the planning data to an ${ }^{192}$ Ir remote afterloader system (Microselectron HDR ${ }^{192}$ Ir; Nucletron, an Elekta company, Elekta AB, Stockholm, Sweden), irradiation has started. The irradiation took approximately $5 \mathrm{~min}$. The applicators were removed after irradiation was complete and the patient was discharged after 2 hours under observation. No complications were reported during the treatment and the brachytherapy was well tolerated by the patient. The patient is regularly followed- up at our affiliated clinics.

\section{Results}

\section{Patient follow-up}

One month after the brachytherapy, it could be observed that the tumor under left neck skin surface shrank obviously with slight radiation-induced skin reaction (grade I), which was characterized by redness and pigmentation. During the follow-up examination in the $7^{\text {th }}$ month after brachytherapy, it was showed that the ipsilateral neck mass disappeared according to the MRI results. The related region of skin surface was fully recovered. At the present 14 months after brachytherapy, treatment-related skin side effect has completely healed. 


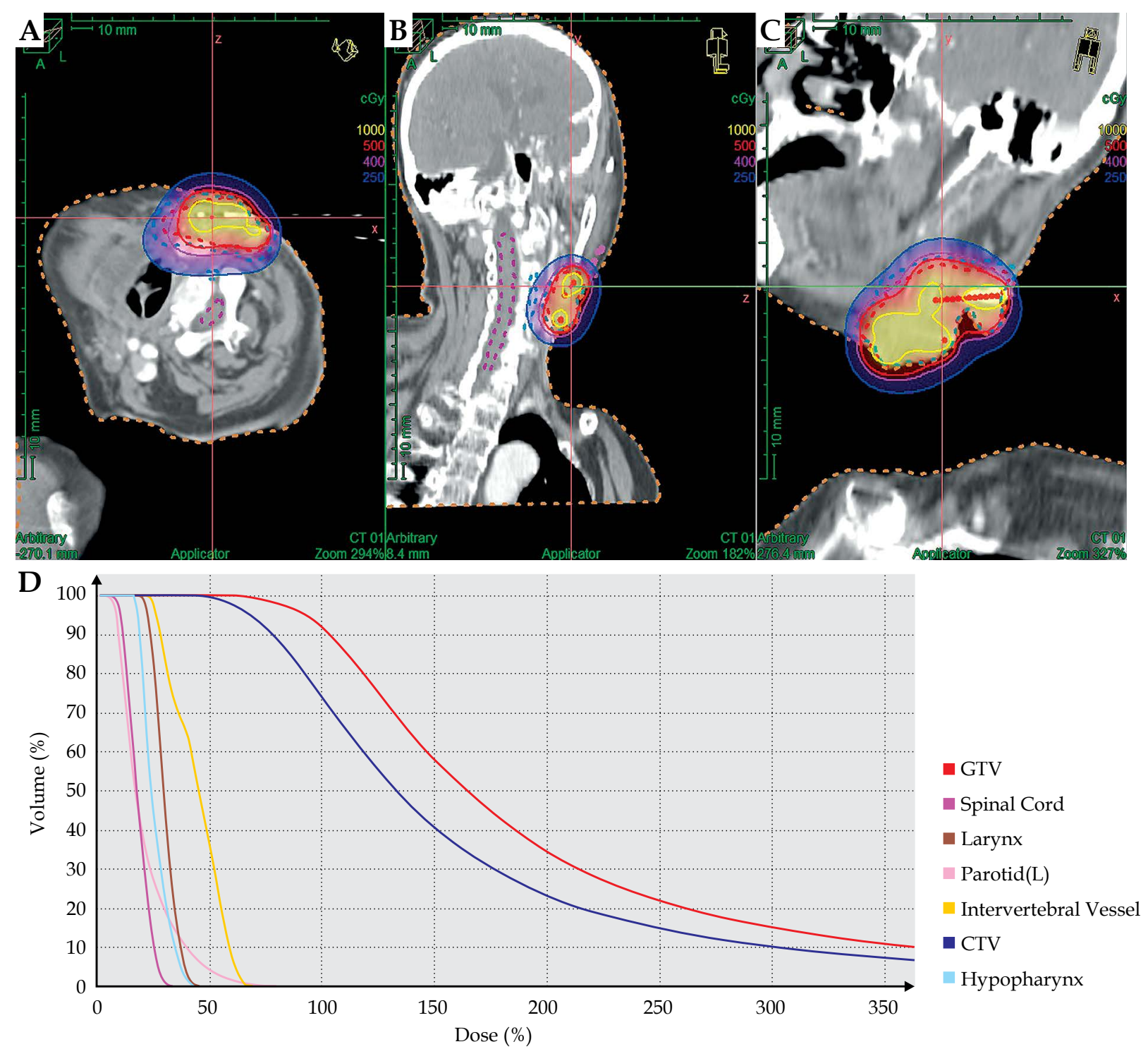

Fig. 4. The dose distribution of (A) horizontal, (B) coronal, and (C) sagittal positions. The red line showed the position of $\mathrm{V}_{100 \%}$, covered gross target volume (GTV) completely. The dose volume histogram (DVH) parameters (D). It showed high dose distribution of GTV and clinical target volume (CTV), and low dose distribution of spinal cord, left intervertebral vessel, larynx, left parotid gland, and hypopharynx

And there are no signs or symptoms of complications and no evidence of recurrence at the site of HDR interstitial brachytherapy (see Figure 5).

\section{Discussion}

Papillary thyroid cancer is the most common but least aggressive histological subtype of thyroid cancer with most of patients having excellent prognosis. However, recent studies have demonstrated increasing incidence of recurrent PTC [6]. Grogan et al. reported a retrospective review of 269 patients with PTC. The results showed that risk factors for recurrence were older age, follicular variant of PTC, T4 tumors, cervical lymph node involvement, metastases, and stage $\geq 4$ a [7]. An observational study by Giordano et al. determined that lateral neck compart- ment ipsilateral to the tumor was the most common site of recurrence with about half of cases appearing in the first 28 months of follow-up [8]. Some treatment strategies, including locoregional and/or systemic therapy, can be considered to treat the recurrent thyroid cancer $[9,10]$. The optimal treatment for refractory recurrence of thyroid cancer is not clearly defined yet. Wolf et al. [11] administered intraoperative radiation therapy, endotracheal hyperthermia, and brachytherapy for advanced thyroid cancer. In another study by Rozdilsky et al. [12], in the treatment of thyroid cancer with interstitial radiotherapy, ${ }^{198} \mathrm{Au}$-comizol was used. Parker et al. evaluated their experience with implantation of ${ }^{125} \mathrm{I}$ seeds in the cerebral mass originated from PTC. They found the cerebral metastases have not recurred, although tumor has 

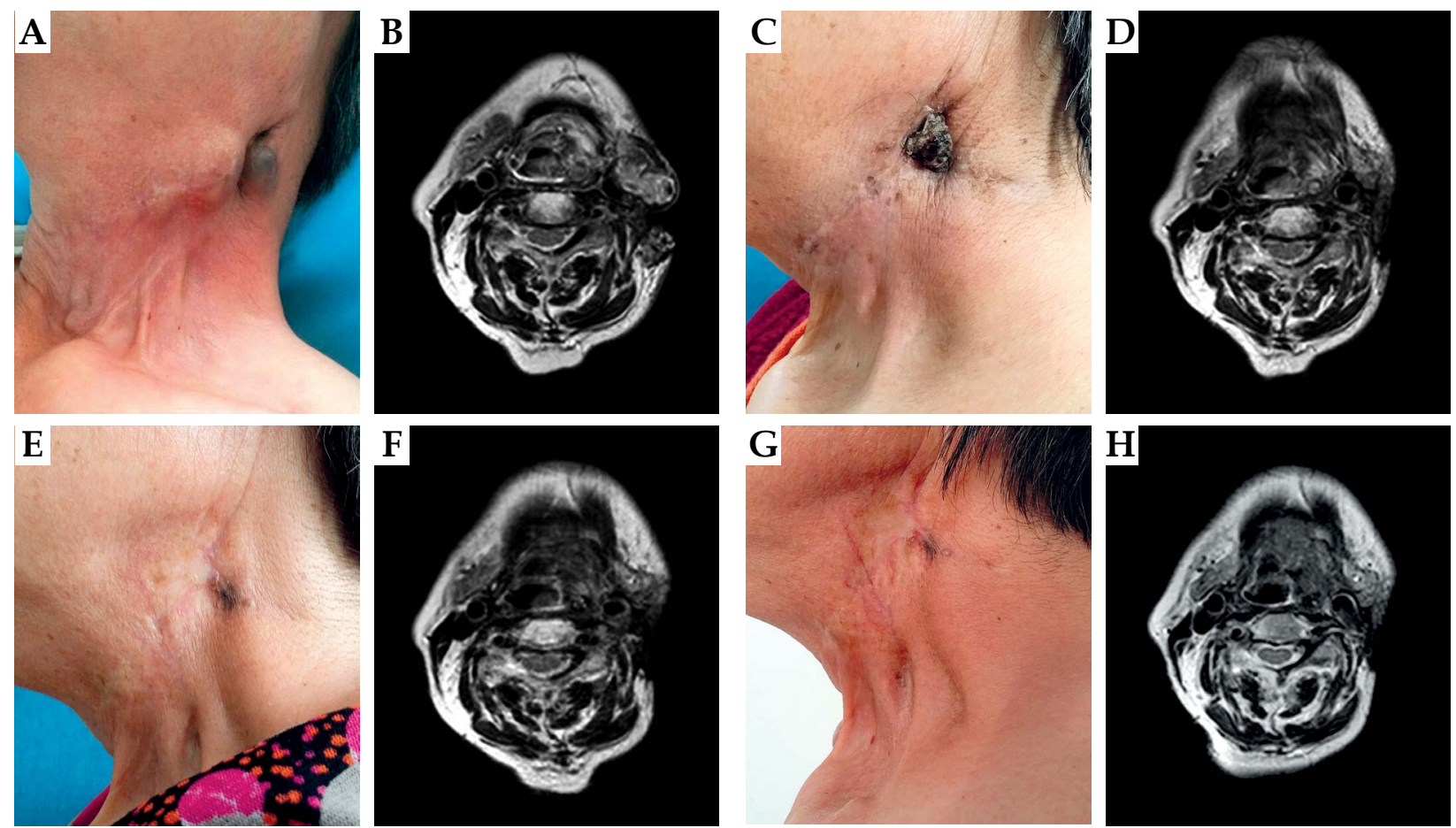

Fig. 5. The left neck (A, B) before, (C, D) 1 month, (E, F) 7 months, and (G, H) 14 months after image guided high-dose-rate interstitial brachytherapy

reappeared in other sites [13]. Kim et al. investigated the feasibility and efficacy of stereotactic body radiotherapy as salvage treatment for cervical recurrence from nonanaplastic thyroid cancer (seven patients had PTC and two had medullary carcinoma) refractory to other modalities. These patients developed nodal recurrence after they received salvage surgery and/or radioisotope treatment for recurrent thyroid cancer. The median follow-up period was 23 months. No local progression was observed in nodes treated by stereotactic body radiotherapy [14]. However, there is little reported in the medical literature concerning the interstitial brachytherapy for PTC recurrence. In the present case, the HDR interstitial brachytherapy with good adherence was used for this patient in order to control the recurrent tumor. Five applicator needles were inserted with the guidance of ultrasound. And the DVH showed that GTV was covered with high dose irradiation but the dosage of spinal cord, left intervertebral vessel, larynx, left parotid gland, and hypopharynx were relative low. Although the radiosensitivity of PTC is not high, the effect after the interstitial brachytherapy was remarkable. The patient with refractory recurrence obtained good locoregional control using the interstitial HDR brachytherapy technique by virtue of image-guidance. The tumor shrank obviously one month later and no serious complications were found in follow-up over 14 months after HDR interstitial brachytherapy. High-dose-rate interstitial brachytherapy provided a superior therapeutic ratio and enabled curative dose treatment with prominent therapeutic enhancement. The results of treatment still need to be monitored and evaluated.

\section{Conclusions}

Although there is ongoing discussion regarding the best management and/or optimal treatment procedure for recurrence of PTC, the image-guided HDR interstitial brachytherapy may be a safe and practicable method. The main benefit of HDR interstitial brachytherapy is that a high dose of radiation can be precisely applied to the tumor while sparing radiation to healthy tissues simultaneously. In select patients, the image-guided HDR interstitial brachytherapy may be a valuable salvage treatment approach for refractory recurrence of PTC.

\section{Acknowledgements}

This study was partially supported by a grant from the National Natural Science Foundation of China (81201737) and Bethune Medical Research Program (201310724, 20160101079JC), Health and Family Planning Commission Foundation of Jilin Province (2014ZC054).

\section{Disclosure}

Authors report no conflict of interest.

\section{References}

1. Hu MI, Vassilopoulou-Sellin R, Lustig R et al. Thyroid and parathyroid cancers. In: Cancer Management: A Multidisciplinary Approach. Pazdur R, Wagman LD, Camphausen KA, Hoskins WJ (eds.). $11^{\text {th }}$ ed. Congregational Ministries Publishing, Louisville 2008.

2. National Cancer Institute. What you need to know about thyroid cancer. National Cancer Institute, Rockville 2012; 6 .

3. Nguyen QT, Lee EJ, Huang MG et al. Diagnosis and treatment of patients with thyroid cancer. Am Health Drug Benefits 2015; 8: 30-40. 
4. Jillard CL, Scheri RP, Sosa JA. What is the optimal treatment of papillary thyroid cancer? Adv Surg 2015; 49: 79-93.

5. Mazzaferri EL, Kloos RT. Clinical review 128: Current approaches to primary therapy for papillary and follicular thyroid cancer. J Clin Endocrinol Metab 2001; 86: 1447-1463.

6. Zhu J, Wang X, Zhang X et al. Clinicopathological features of recurrent papillary thyroid cancer. Diagn Pathol 2015; 10: 96.

7. Grogan RH, Kaplan SP, Cao H et al. A study of recurrence and death from papillary thyroid cancer with 27 years of median follow-up. Surgery 2013; 154: 1436-1446.

8. Giordano D, Frasoldati A, Kasperbauer et al. Lateral neck recurrence from papillary thyroid carcinoma: Predictive factors and prognostic significance. Laryngoscope 2015; 125: 2226-2231.

9. Grant CS. Recurrence of papillary thyroid cancer after optimized surgery. Gland Surg 2015; 4: 52-62.

10. Meadows KM, Amdur RJ, Morris CG et al. External beam radiotherapy for differentiated thyroid cancer. Am J Otolaryngol 2006; 27: 24-28.

11. Wolf G, Kohek P, Geyer E et al. Intraoperative radiation therapy, endotracheal hyperthermia, and IR-192-brachytherapy in patients with advanced thyroid cancer. Acta Med Austriaca 1996; 23: 76-79.

12. Rozdilsky SI, Tkacenko GI, Vasiljev LJa et al. Interstitial radiotherapy of thyroid cancer using 198Au-comizol. Radiobiol Radiother (Berl) 1988; 29: 729-733.

13. Parker LN, Wu SY, Kim DD et al. Recurrence of papillary thyroid carcinoma presenting as a focal neurologic deficit. Arch Intern Med 1986; 146: 1985-1987.

14. Kim JH, Kim MS, Yoo SY et al. Stereotactic body radiotherapy for refractory cervical lymph node recurrence of nonanaplastic thyroid cancer. Otolaryngol Head Neck Surg 2010; 142: 338-343. 